liquid remained in contact with polyethylene or nylon connecting tubes. These results may have direct practical importance on industrial process problems, where polyethylene pipes become blocked by masses of slime-forming bacteria attached to the walls, and in the storage of non-sterile solutions in containers made of these materials.

The use of opercular bones to estimate the age and past growth of pike has been further investigated by Dr. Winifred Frost and Miss Charlotte Kipling, who concluded last year that growth can be calculated accurately by simple proportion from measurements of the annual rings, making an arbitrary choice by inspection of a centre from which to make the measurements. Since the rings near the centre are not always clearly visible, the method does not give reliable estimates of growth in the early years of life. This difficulty has been overcome by the use of theoretical growth-tables derived from Ford's growth formula, using data from Windermere calculated for slow-, medium-, fast- and very fast-growing fish for the two sexes separately. By comparing the growth in later years of any individual fish with these tables, it is usually possible to assign it to one of the growthrate categories. This makes it possible to infer its true age and so to decide whether the first discernible ring represents its first, second or third winter.

D. F. Westlake has measured the rate of production of oxygen by Ranunculus spp. in a closed, static system, at various light intensities. The highest gross rate of oxygen production found for lightsaturated plants was about $10 \mathrm{mgm}$. of oxygen $\mathrm{hr}$./gm. (dry weight) at $15^{\circ} \mathrm{C}$. Light-saturation was approached at 10,000 lux, and both this and the maximum rate of oxygen production are lower than generally reported in the literature on higher plants. Possibly this is the result of using static conditions for the experiments, and apparatus for determining oxygen production in flowing conditions is being developed.

\title{
MECHANICAL ENGINEERING RESEARCH LABORATORY
}

\section{REPORT FOR 1957}

$\mathrm{T}$ WE report on the work of the Mechanical Engineering Research Laboratory for $1957^{*}$ makes fascinating reading, and contains much that will interest any engineer and many physicists.

The chairman of the Engineering Research Board directs attention in his report to the difficulty of getting the results of the Laboratory's work both disseminated and applied. Most engineering scientists have yet to learn that a paper which may lead in due course to scientific honour is unlikely to be understood by many practising engineers, however high their theoretical attainments; the work must also be published as a straightforward physical description, written in lucid English and devoid of mathematics if it is to be read and applied. Sir Charles Inglis used to state this truth regularly in his lectures; few have learned the lesson.

The proportion of scientific and experimental staff is 160 out of a total of 444 . This seems high. Does the Laboratory suffer from the difficulty common in

* Department of Scientific and Iudustrial Research. Mechanical Engineering Research 1957: Report of the Mechanical Engineering Research Board, with the Report of the Director of Mechanical Engineering Research. Pp. iv $+63+4$ plates. (London: H.M. Stationery Ottice, 1958.) 4s. $6 d$. net.
Government and university laboratories of being hamstrung financially in providing adequate ancillary and office staff?

In recommending a rapid expansion of the Laboratory, the problem of how it, the universities and the colleges of advanced technology are all to be staffed must arise. In Germany, most of the work similar to that done at the Mechanical Engineering Research Laboratory is done in institutes of the Technische Hochschulen. Might it not be better to give the colleges of advanced technology similar institutes?

While it is clear why some of the research, both fundamental and applied, is being done nationally, it is surprising to find that fundamental work on greases has not already been undertaken on an adequate scale by private industry. When such gaps are found, is it not desirable that they should be investigated where the staff concerned can also be available for part-time teaching ?

Nevertheless, the report describes the development of fine facilities and the accomplishment of much excellent work. Many firms should read it and send members of their staff to see what is being done.
D. B. WELBOURN

\section{TILT CRITERIA AND DIRECTION OF ROTATION OF SPIRAL GALAXIES}

G DE VAUCOULEURS has a paper with the J. above title in the Astrophysical Journal, 127, 2 ; March 1958, in which he presents a review of the observational evidence on the direction of rotation of the spiral pattern in ordinary and barred spirals. A growing body of evidence suggests that the formation of spiral structure is governed by the large-scale magnetic and hydrodynamical properties of the interstellar medium in rotating galaxies, and according to current ideas, dust or 'smoke' particles may form out of the gaseous medium and induce the condensation of gas clouds into stars or groups of stars. The author of the paper has discussed the morphology of spiral structure and its bearing on the large-scale instantaneous distribution of gas and dust in galaxies (Hand. Phys., 53), and in the present paper he deals with the systematic motion of the interstellar substratum on the largest scale in a galaxy, in other words, with the general rotation of the spiral pattern. The previous investigations of those who have devoted their attention to this subject-Baade, Hubble, Lindblad, Stebbins, Zwicky and others-are referted to, and the reasons for the divergences in the interpretations are analysed. The discussion is recapitulated in Table 1, p. 499, which consists of eleven columns (the symbols used in these are explained at the end of the table and also on the previous page) and the conclusions are briefly as follows. 